\title{
1 Medical applications of silicon photomultipliers
}

2 Maria Giuseppina Bisogni, Alberto Del Guerra(*), Nicola Belcari

3 Department of Physics "E.Fermi", University of Pisa and INFN, Sezione di Pisa

5 Abstract

6 SiPMs are becoming the reference photodetectors in many fields. In medicine there are slowly

7 replacing photomultiplier tubes and avalanche photodiodes in medical imaging and in PET in

8 particular. In this paper a broad overview of the current applications of SiPM in Medicine is

9 presented. The major fields where the SiPMs are used, namely PET/MR and hadrontherapy are

10 discussed at length.

$12 \quad(*)$ Corresponding author

13 Email: alberto.del.guerra@unipi.it

14

\section{1. Introduction: from photomultipliers to SiPMs}

16 The photomultiplier tube (PMT) has been the most used device since the invention of NaI (Tl) by

17 Hofstadter 1948 [1] to collect the light produced by a $\gamma$-ray interaction within a scintillator. This

18 was due to the photocathode high quantum efficiency (QE of about 15-25\%) and to a high signal

19 amplification factor of about $10^{6}$ that allowed using simple off-the-shelf electronics to collect 
1 data. These properties and simplicity were essential for their application to the medical field and

2 in particular for the imaging of $\gamma$-rays emitted by radiotracers in nuclear medicine, for planar

3 scintigraphy with the Anger camera and for Single Photon Emission Computed Tomography

4 (SPECT) and Positron Emission Tomography (PET) [2]. In PET application the fast time

5 response of the PMT was also an extremely important property for the reduction of the random

6 coincidences rate and very recently for the so-called Time of flight PET (TOFPET) [3]. The

7 original coupling in PET was one to one, i.e. one crystal coupled to one PMT of typical cross

8 section diameter of 2" inches. In 1986 Casey and Nutt [4] introduced the scheme of the "block

9 detector" based on a combination of four PMTs coupled to a single crystal block with a structured

10 cutting that determined the advent of PET to the clinical stage. In early 2000, with the

11 development of molecular imaging based on small animal studies, the spatial resolution of the

12 clinical PET scanners at the time did not appear to be satisfactory for obtaining a quantitative

13 imaging on rats and mice, where the spatial resolution required was about 1-2 $\mathrm{mm}$. This solicited

14 the use of Position Sensitive PMTs (PSPMT) coupled to pixelated scintillators, where the fine

15 positioning was obtained with an Anger camera logic.

16 Such a family of position sensitive tubes was originally characterized by a large detection area

17 (up to 5 inches diameter), but the round geometry and the peripheral dead zone (1 cm or more)

18 prevented these tubes to be tightly assembled in arrays to cover larger surfaces. A successful

19 example of such a use of the Hamamatsu R2486 is the small animal PET scanner (YAP-PET)

20 built in 1998 [5]. Then the second PSPMT generation was built based on metal channel dynode

21 for charge multiplication by which the intrinsic spatial resolution could reach $0.5 \mathrm{~mm}$ FWHM.

22 The first version of these new tubes (model R7600-C8) was employed for the construction of the

23 MicroPET at UCLA [6]. Finally, the third generation of Multi Anode PMTs by Hamamatsu,

24 namely H8500 (64 channels) and H9500 (256 channels), was developed with an external size of

$2552 \mathrm{~mm} \times 52 \mathrm{~mm} \times 28 \mathrm{~mm}$ and an active area of $49 \mathrm{~mm} \times 49 \mathrm{~mm}$. However, researchers still 
1 looked for alternative solutions able to provide the same (or better) intrinsic resolution in a more

2 compact and customizable form factor that can be tailored, e.g, for the design of original detector

3 geometries [7], for the construction of depth of interaction (DOI) capable detectors based on

4 monolithic crystals [8] or for pushing the intrinsic resolution beyond the state of the art [9].

5 In early 2000, the hybrid systems were coming available with the SPECT/CT and PET/CT that

6 were combining the anatomical (CT) and functional (SPECT and PET) information on the patient.

7 Furthermore, there was a great interest in taking advantage of the exquisite resolution of MRI for

8 low density tissue to be combined with the SPECT/PET functional information. This triggered

9 the research in building such hybrid scanners especially PET/MR combined in a single device

10 [10]. With the PMT technology it was not possible to make such integration because of the

11 incompatibility of the PMTs with magnetic fields [11]. In fact, a magnetic field as low as $\approx 3 \mathrm{mT}$

12 already produces significant variation in gain and distortion in the path and multiplication of the

13 electron within the vacuum tube [12]. Hence it is clear that PMTs cannot operate at the magnetic

14 field strengths typical of the MR clinical devices (e.g. $1.5 \mathrm{~T}$ and $3 \mathrm{~T}$ ).

15 Solid-state photodetectors were invented in the late 1940s [13] with the first p-n junction in silicon

16 and the production of PIN diodes that were later used in radiology. The development of the

17 avalanche photodiodes (APD) in 1950s [14] and later on of the Geiger-mode avalanche

18 photodiodes, also named single photon avalanche diode (SPAD), lead to a substantial

19 improvement in sensitivity and allowed the detection of the single photon. Later on, thousands of

20 SPADs have been assembled in arrays of few millimeters squared (currently named silicon

21 photomultiplier, SiPM) [15] and several SiPMs have been arranged in a matrix structure so as to

22 cover large areas [16]. The high internal gain of SiPMs, together with other features peculiar of

23 the silicon technology such as compactness and speed, since the beginning promoted SiPM as the

24 principal photodetector competitor of photomultipliers in PET [17], [18]. Finally, their full 
1 compatibility with magnetic fields enabled the construction of hybrid PET/MR systems, allowing

2 the full integration of the two imaging techniques.

3 The topology of a SiPM based detector in Nuclear Medicine applications varies according to the

4 performance (e.g., intrinsic spatial resolution, sensitivity, depth of interaction information, time

5 resolution, and so on) one wants to optimize most for the particular application: matrix of

6 scintillator coupled to a matrix of SiPM (Figure 1a), monolithic scintillator coupled to a matrix

7 of SiPM (Figure 1b), matrix or monolithic scintillator coupled to two opposite matrices of SiPMs

8 (Figure 1c and Figure 1d). The readout of the SiPM matrix can be done individually via an ASIC

9 or by a resistive chain readout. The size of a detector module is usually of the order $5 \mathrm{~cm} \times 5 \mathrm{~cm}$,

10 that is a reasonable compromise between count rate capability and data handling.

11

a

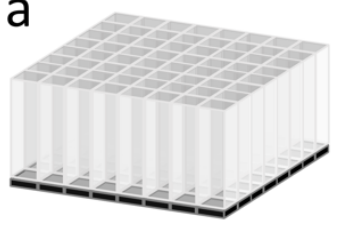

b

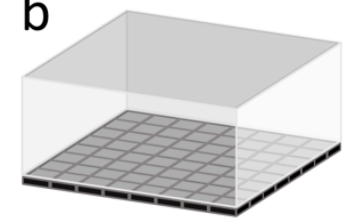

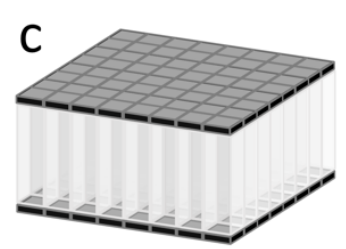

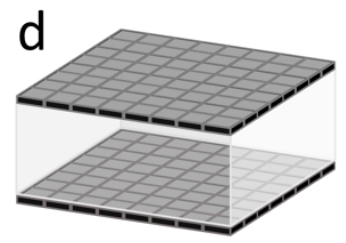

12

13

Figure 1: Typical arrangements of SiPMs for the readout of scintillators. a) One side readout of pixelated matrices,one-to-one coupling or light sharing, b) one side readout of a monolithic scintillator block, c) and d) dual side readout of pixelated and monolithic blocks, respectively.

The paper is organized as follows: after the introduction that shows why the SiPM are replacing the PMTs in Medical Imaging, in the following sections there are presented most of the SiPM based applications: small animal scanners for molecular imaging (Section 2), the new clinical PET scanners, including TOFPET (Section 3) and the Hybrid PET/MR and SPECT/MR scanners (Section 4). In section 5, the recent applications of SiPM in PET and Compton camera in for hadrontherapy are discussed. Finally, the intra-operative probes applications are presented in Section 6. The Conclusions and Future Outlook section completes the paper. 


\section{2. Small animal PET Imaging}

2 Small animal (or preclinical) PET is a research field where some of the typical challenges of PET

3 imaging, i.e., high resolution, high sensitivity and count rate performance are taken to the extreme

4 level. In particular, spatial resolution is pushed towards the ultimate limit of PET imaging [2]. For

5 this reason, the intrinsic spatial resolution of the photodetector plays a relevant role. Since the

6 early years of development of small animal PET systems, solid state photodetectors were

7 considered a valuable alternative to standard photomultiplier tubes. With the advent of high

8 granularity APD arrays, a number of detector designs were proposed for the readout of finely

9 pixelated scintillator matrices so as to build high resolution preclinical PET systems [19,20].

10 However, the widespread adoption of this solution was limited by the complexity of the data

11 acquisition electronics. In fact, for the limited gain of APDs $\left(\sim 10-10^{2}\right)$, each output signal requires

12 a high amplification before acquisition, thus resulting in a large number of channels to be

13 independently preamplified and acquired. Then, the most successful preclinical PET systems on

14 the market such as the Siemens Inveon [21] or the Mediso nanoPET [22] families were all based

15 on some sort of high granularity multi anode photomultiplier tubes coupled to pixelated matrices,

16 while other systems, such as the Bruker Albira [23], were implementing monolithic crystals.

17 Although the replacement of PMTs with SiPM was boosted by the need of photodetectors able to

18 work in a magnetic field and then suitable for the construction of integrated PET/MR systems

19 (see section 4), the characteristics of SiPMs make them the ideal replacement of multi anode

20 PMTs for the design of small animal PET systems with improved performance with respect to the

21 present generation.

22 Today, SiPM matrices are available with pitches similar to multi anode PMTs. For example, 23 Hamamatsu offers Multi Pixel Photon Counter (or MPPC, the Hamamatsu brand name for SiPM) 24 arrays with SiPM size down to $2 \mathrm{~mm}$ on a $2.2 \mathrm{~mm}$ pitch (e.g., the S13361-2050AE-04 model) 
1 packaged in an array of $4 \times 4$ elements. For comparison, multi anode photomultiplier tubes are

2 available today with similar channel size. For example, the series R7600-200-M64 by Hamamatsu

3 has 64 independent readout anodes arranged in a matrix of $8 \times 8$ channels with a $2 \mathrm{~mm}$ pitch,

4 while the more popular Hamamatsu H13700 series has $256(16 \times 16)$ independent channels with

5 a $3.0 \mathrm{~mm}$ pitch.

6 It is clear then that it is not the high intrinsic spatial resolution achievable with small pitch SiPM

7 matrices the killer feature for replacing multi anode PMTs in preclinical PET application, but its 8 compactness. The preclinical PET system $\beta$-CUBE by Molecube (Gent, Belgium) is a typical

9 example of this rationale. This system is designed to offer PET only imaging of mice and rats in

10 a compact benchtop system format. Thanks to the compactness of SiPMs, the whole system has

11 a footprint of $54 \mathrm{~cm} \times 54 \mathrm{~cm}$ with a weight of $78 \mathrm{~kg}$ while offering performance at the state of the

12 art [24]. To achieve this goal each detector module features a $64(8 \times 8)$ channels MPPC array with

$133 \mathrm{~mm}$ pixel size for the readout of a $25.4 \times 25.4 \times 8 \mathrm{~mm}$ thick monolithic LYSO scintillator.

\section{Clinical PET and TOF-PET}

In time-of-flight (TOF) PET the measurement of the time difference in the detection of the two annihilation photons is used to reduce the uncertainty in the determination of the position of positron annihilation along the line-of-response between two detectors. The ultimate effect of the introduction of the TOF information in PET image reconstruction is an effective gain in sensitivity that is proportional to $1 / \mathrm{CTR}$, where CTR is the coincidence time resolution. The introduction of fast scintillators with high density such as LSO:Ce or LYSO:Ce have made it possible to develop clinical whole body TOF-PET systems. As of today, clinical TOF-PET/CT systems have a CTR below 600 ps [25]. 
1 The photosensor used in a TOF-PET detector has to be capable of deriving the time information

2 with an intrinsic time resolution at the limits imposed by scintillators, i.e., not introducing any

3 further time degradation. Dealing with a low light situation, the photosensor should be extremely

4 sensitive with single photon detection capability and ultra-fast responding (in the ps range).

5 SiPMs are well suited for this purpose with timing performance similar to fast PMTs.

6 For example, a single photon time resolution below 100 ps FWHM [26] has been obtained

7 irradiating in coincidence two SiPMs with a laser source, while a standard deviation of about 20

8 ps has been achieved by increasing the number of micro-cells triggered simultaneously.

9 A potential advantage of SiPM over fast PMTs for TOF-PET is the capability of providing more

10 information on the time distribution of the emitted scintillation light. For instance, in TOF-PET a

11 high-speed trigger network and an integrated TDC can be implemented to extract the time

12 information [27].

13 The first clinical TOF-PET/CT system based on SiPMs, the Vereos Digital, was developed by 14 Philips. It is based on digital SiPM (dSiPM) photodetectors [28]. In this case a one-to-one 15 coupling of LYSO crystals with the surface of $4 \times 4 \mathrm{~mm}^{2}$ to dSiPMs of about the same size was 16 implemented thus providing a high count-rate capability and a CTR $<316$ ps.

17 Recently, a whole body PET/CT system using SiPMs, named Discovery MI, has been launched 18 by GE Healthcare [29], while Siemens Healthineers announced in 2017 a new PET/CT system 19 (named Biograph Vision) featuring $3.2 \mathrm{~mm}$ pitch LSO crystal matrices read out by SiPMs. The 20 fact that all the three major players in the molecular imaging sector are implementing SiPMs in 21 their more advances TOF-PET/CT systems has represented a milestone in the history of PET 22 detectors, as it assumes the overall superiority of SiPMs over PMTs, both in cost efficiency and 23 performance [30]. 


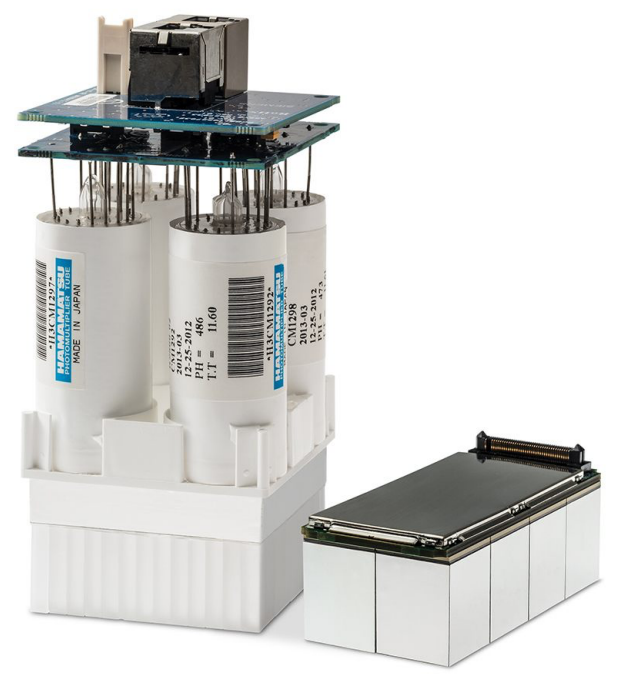

1

Figure 2: Picture showing the comparison of PET detectors based on PMTs (left) and on SiPMs (right) in their implementation by Siemens Healthineers (picture from https://www.healthcare.siemens.com/molecular-imaging/pet-ct/biograph-vision\#CLINICAL_USE accessed August 22, 2018).

6

7 Although the capability of near 100 ps time resolution was demonstrated in laboratory, the

8 availability of PET systems with such a performance at system level are still not available. Further

9 research in new scintillating materials and data acquisition systems able to provide a minimal

10 degradation of the intrinsic timing performance of the photodetector plus scintillator combination

11 is necessary. As for the SiPM itself, an improvement of the PDE [26] as well as the integration in

12 SiPM of multiple TDCs in CMOS technology [31] would also significantly contribute to the

13 improvement of the CTR in future TOF-PET systems.

15 4. Hybrid scanners: PET/MR and SPECT/MR

16 The advent of the PET/CT Hybrid scanners in the clinical applications triggered the idea of

17 developing PET/MR and SPECT/MR scanners, to obtain a fusion of the exquisite anatomical $-8-$ 
1 MRI information with the functional information given by PET and SPECT. Because of the

2 incompatibility of the PMTs with the magnetic field, after the first attempt to operate the two-

3 modality scanner sequentially (the so-called tandem configuration, see Fig. 2, top left) it was clear

4 that to have a simultaneous hybrid system it was necessary to use semiconductor photodetectors,

5 initially APD and then SiPM. The research concentrated on PET/MR [10] and two possible

6 configurations: i) the "insert" solution where the PET ring was insertable/removable from an

7 existing MR (Figure 3, top right), ii) the "fully integrated solution" (Figure 3, bottom), where the

8 MR and PET where built from the beginning as a single instrument.

9

10

11 Figure 3: Artistic cross view of various PET/MR configurations, a) Tandem: the patient shares the same bed that is sequentially moved from MR to PET scanner; b) INSERT: the PET scanner is inserted in a preexisting MR system, c) the PET/MR is built as an integrated scanner [10]

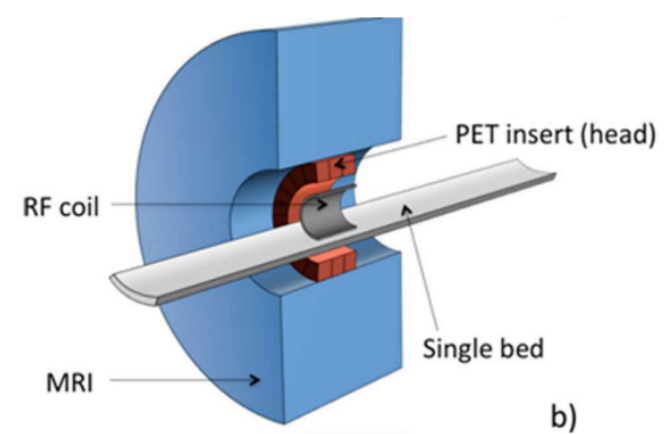

a)

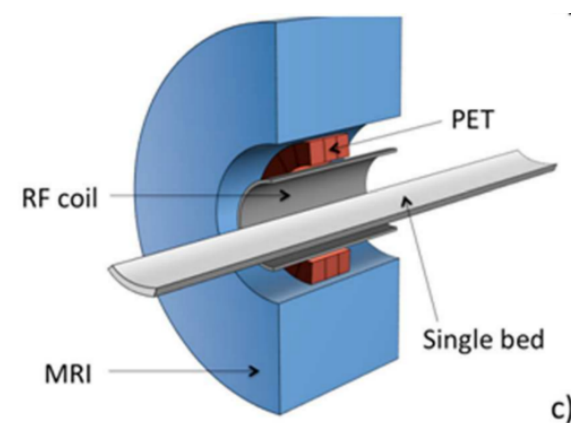

c) 
1 One of the first prototypes for a simultaneous PET/MR scanner developed by in 2006 by the

2 Oxford group used the "MicroPET" small animal scanner inserted within a split-field magnet

3 [32]. The light from the scintillator ring that was inside the magnet was conveyed far away to the

$4 \quad$ PMTs by means of long light guides. In this way the PMTs could operate in the stray magnetic

5 field, but the signal was deteriorated because of the light absorption within the guide. In the same

6 years, the first PET insert for simultaneous PET/MR imaging was build using APDs as

7 photodetectors [33] directly plugged on a board hosting the frontend electronics. With the

8 introduction of SiPMs a number of PET inserts for PET/MR has been developed. These systems

9 feature different solutions for taking the signals out of the PET detectors with the aim of

10 mitigating the mutual interference between PET and MR. The solutions range from using optical

11 fiber bundles [34] or coaxial cables [35] to convey the signal to the electronics, positioned outside

12 of the magnet, or by using MR compatible frontend electronics and power supply [36]. Preclinical

13 PET inserts based on SiPM are also now available on the market by MR Solutions and Bruker.

14 The Siemens Biograph mMR based on APD photodetector technology was the first whole-body

15 simultaneous PET/MR clinical scanner [37]. The SiPMs were shortly suggested as an attractive

16 alternative to the APDs, the major advantages of SiPM being a much higher gain (at least a factor

$1710^{3}-10^{4}$ ), a much faster rise time (about a factor 3 ) and a lower supply voltage (about a factor 10 ).

18 The first attempts were made by Nassalki et al. [38] and by K.Jo.Hong [39]. The first PET/MR

19 commercial system based on the SiPM technology was produced by GE Healthcare. The GE

20 SIGNA PET/MR, has a transversal FoV of $60 \mathrm{~cm}$ and an axial one of $25 \mathrm{~cm}$. The PET system is

21 combined with a 3T magnet. The PET scintillator is LFS, with dimensions of $4 \mathrm{~mm} \times 5.3 \mathrm{~mm} \times$

$2225 \mathrm{~mm}$. The scintillator matrix is coupled to analog SiPMs combined with UV transparent light

23 guides for light sharing to reduce the number of readout channels. It has TOF capability (390 ps 
1 CTR for the whole system), an energy resolution of $10.5 \%$ and a spatial resolution of $4.2 \mathrm{~mm}$.

2 The sensitivity at the center of the Field of View is $22 \mathrm{kcps} / \mathrm{MBq}$ [40].

3 In 2012, the first simultaneous PET/MR preclinical device equipped with PET detector modules

4 based on digital SiPMs was presented [41,42]. A Single Detection Module (SDM), based on

5 LYSO arrays $\left(30 \times 30\right.$ crystals of $1 \times 1 \times 12 \mathrm{~mm}^{3}$ each $)$ is connected one-to-one to Digital SiPMs

6 by Philips Digital Photon Counting; $3 \times 10$ of these SDMs form a ring and 3 rings are juxtaposed

7 to build the preclinical PET/MR insert Hyperion II [43,44]. It covers an axial FoV of $20.96 \mathrm{~mm}$

$8 \times 96 \mathrm{~mm}$ transaxial, with an energy resolution of $11.4 \%$, a CTR of $213 \mathrm{ps}$ and a 3D spatial

9 resolution of $0.73 \mathrm{~mm}$.

10 In parallel to whole-body PET/MR clinical systems, several groups started developing organ 11 specific PET/MR: brain has been the privileged target. One of the active groups in this field was 12 the Forschungszentrum Julich group that in collaboration with Siemens implemented a 9.4T MRI 13 combined with the BrainPET based on APD [45], to obtain the first Brain PET/MR.

14 The increasing interest in brain imaging is not limited to neuro-oncology and neuro-inflammation, 15 but is extending to the field of neuroscience and psychiatry disorders [46]. This is also evident 16 from the specific call within the $7^{\text {th }}$ Framework Programme, (HEALTH.2013.2.2.1-2: 17 Development of effective imaging tools for diagnosis, monitoring and management of mental 18 disorders), where two projects for the development of PET/MRI scanners were funded.

19 The first one, MINDVIEW [47] aimed to build a SiPM based insert for a $3 \mathrm{~T}$ MR. The 20 technological challenge for PET was to use a monolithic crystal read by one matrix of SiPM, so 21 as to reach an intrinsic spatial resolution below $1 \mathrm{~mm}$. The scanner is made of three rings of 20 22 detector modules per ring Figure 4. The internal PET diameter is $33 \mathrm{~cm}$ with a transaxial FoV of 
$124 \mathrm{~cm}$ and an axial field of view of $15.2 \mathrm{~cm}$. The monolithic crystal is LYSO $(5 \mathrm{~cm} \times 5 \mathrm{~cm} \times 2$

$2 \mathrm{~cm})$ seen by a matrix of $12 \times 12$ SensL $\operatorname{SiPM}(3 \mathrm{~mm} \times 3 \mathrm{~mm}$ each). The SiPM readout scheme is

3 done by rows and columns, thus reducing the number of channel output. The measured intrinsic

4 spatial resolution obtained with Depth of Interaction (DOI) correction varies between 0.7 and 1.7

$5 \mathrm{~mm}$. The sensitivity is $2.7 \%$ for a point source at the center of the field of view. The PET scanner

6 was successfully tested within the MR system of the mMR PET/MR system at Klinikum Recths 7 der Isar in Munich [48].

8

9

10

11

12

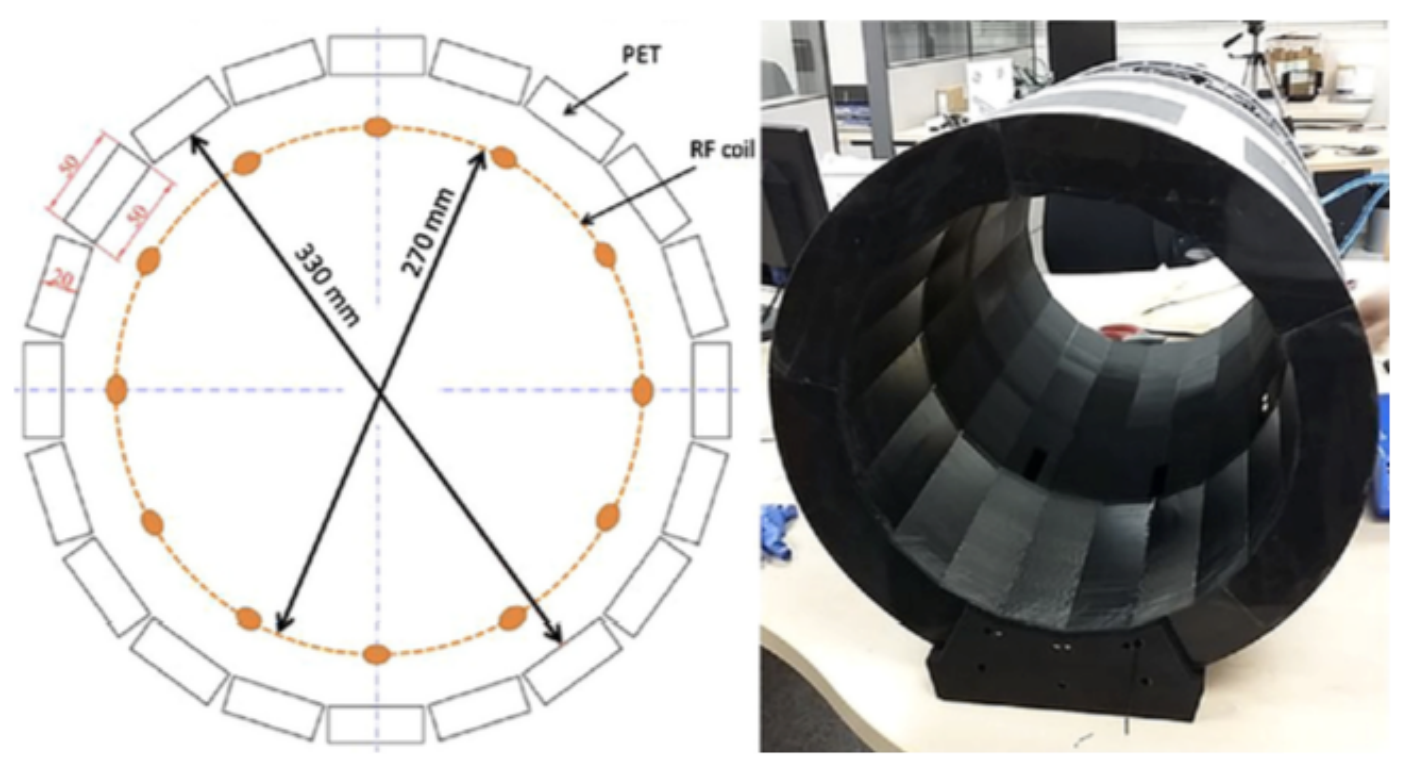

Figure 4: Sketch of the MINDVIEW PET scanner (left); MINDVIEW PET scanner on the bench [48]

The other FP7 funded project, TRIMAGE [49], represents the first effort toward the development of a full brain PET/MR system [50] with the additional capability of accommodating an MR compatible ElecroEncephaloGraphy (EEG). TRIMAGE aims to create a trimodal, cost-effective 
1 imaging tool consisting of PET/MR/EEG, using cutting edge technology with performance

2 beyond the state of the art (Figure 5).

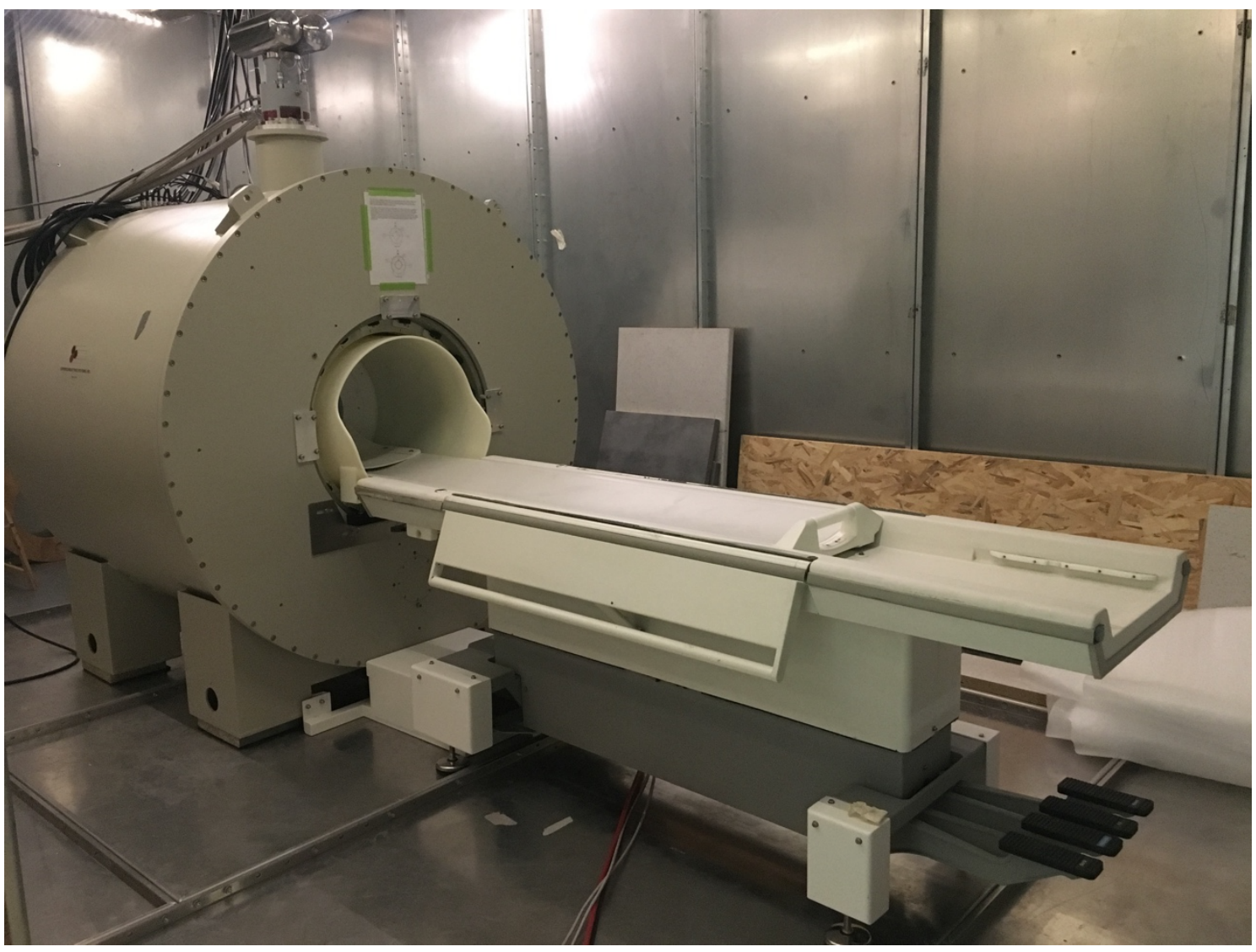

4 Figure 5: Picture of the TRIMAGE system in its preliminary installation setting.

5

The MR is a superconducting 1.5T magnet without any cryogenic liquid. The PET insert has a transaxial FoV of $31 \mathrm{~cm}$. It consists of 54 modules of $5 \times 5 \mathrm{~cm} 2$, arranged in 18 rectangular detectors of $5 \times 15 \mathrm{~cm} 2$ to form a cylindrical geometry. Each module is made of four submodules of $2.5 \times 2.5 \mathrm{~cm} 2$; each submodule is made by a dual-staggered layer of LYSO matrices so as to estimate DOI. Front and back layer are $8 \mathrm{~mm}$ and $12 \mathrm{~mm}$ thick, respectively. The photodetectors for each sub-module consists in 2 arrays of $4 \times 8$ NUV SiPMs by Advansid and a dedicated 64 channel ASIC [51] (Figure 6). 

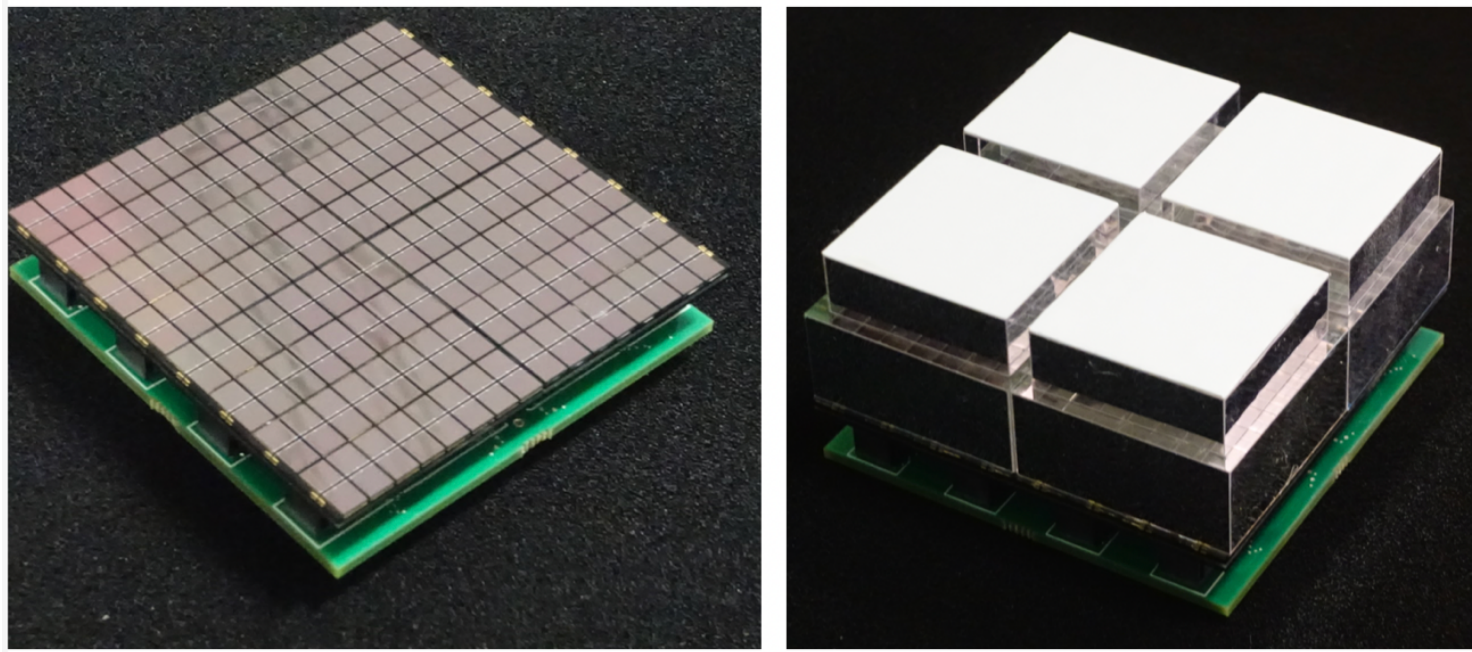

2 Figure 6: Left, picture of the SiPM array of the TRIMAGE PET detectors. It consists of $8(4 \times 2)$ 3 matrices of SiPMs manufactured by AdvanSiD, Italy. Each matrix has $32(8 \times 4) 3 \mathrm{~mm} \times 3 \mathrm{~mm}$ 4 SiPMs. Right, picture of the PET detector module, featuring four dual-staggered layer of 5 LYSO matrices. The board below the detector module hosts four readout ASICs.

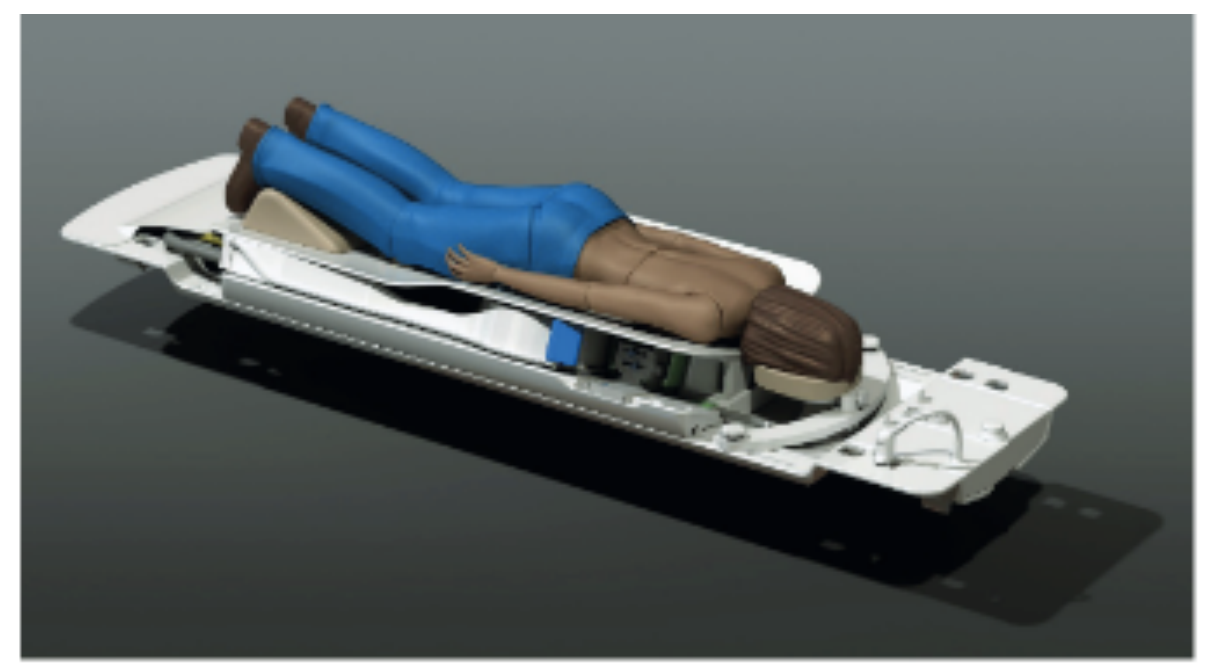

8 Figure 7: Schematic of the HYPMED device. The patient is a prone position and both breasts can be 9 independently accessed by the two PET rings towards the feet of the patient (from www.hypmed.eu, 10 accessed 16 August 2018)

11 The preliminary performances of the system are: a spatial resolution that varies between 2.34 and

$123.66 \mathrm{~mm}$, a sensitivity of $61 \mathrm{cps} / \mathrm{kBq}$ for a source at the center of the field of view, an energy 
1 resolution of about $17 \%$ and a CTR of 420 ps between two single crystals and about $600 \mathrm{ps}$

2 between two entire sub-modules.

3 Alongside with brain dedicated PET/MR scanners there are projects for breast dedicated

4 PET/MR. A full simulation of a PET insert to be integrated in a breast MRI coil was made by

$5 \quad$ Liang and Peng [52].

6 More recently, a Horizon2020 project (HYPMED) has been funded to build a PET/MR breast

7 insert [53]. The PET insert based on SiPM is expected to reach unprecedented imaging of breast

8 cancer with high resolution/ultra-high sensitivity (Figure 7).

9 Hybrid systems that combine SPECT and MR and are using SiPMs are rare, since the technology 10 in SPECT has evolved toward CdTe and CZT semiconductors as direct conversion/detection 11 devices for SPECT $\gamma$ rays. In 2013 the EU-FP7 program funded the project (INSERT) that aims 12 to develop an integrated SPECT/MRI breast system. The SPECT detector is bases on monolithic 13 CsI scintillator of $8 \mathrm{~mm}$ thickness, coupled to an array of 144 SiPMS (RGB-HD type by FBK, 14 Italy) read and multiplexed by a 36 channel ANGUS ASIC. A fundamental part of any SPECT 15 device is the collimator. The INSERT consortium is building both a preclinical and a clinical 16 prototype. The first one has a SPECT detector with a transaxial FoV of $15.6 \mathrm{~mm}$, an axial FoV of $1716 \mathrm{~mm}$ and a multipinhole collimator (nine holes of $0.8 \mathrm{~mm} \times 0.8 \mathrm{~mm}$ ) made of tungsten powder 18 and epoxy resin. The clinical prototype will have a much larger field of view and the collimator 19 will be a multislit-slat geometry. The measurements on the preclinical prototype [54] have given 20 an energy resolution of $14 \%$ for ${ }^{99 \mathrm{~m}} \mathrm{Tc}$ and an intrinsic spatial resolution of $1.1 \mathrm{~mm}$ at $122 \mathrm{keV}$. 


\section{5. Hadrontherapy}

2 Hadrontherapy is a cancer therapy using ions (mainly protons and carbon ions) for the treatment

3 of solid and radioresistant tumours. At the end of 2017 there were 72 centres operating worldwide

4 and another 68 are in construction or in the planning stage, most of which are proton centres.

5 More than 170,000 patients were treated in the time span between 1954 and 2016 (around 150,000

6 with protons and 21,000 with carbon ions [55]).

7 Hadrontherapy is characterised by the depth dose curve with a peak (Bragg peak) at the end of

8 the particle path. The utility for cancer therapy was recognised for the first time by R. Wilson in

91946 [56]. The total energy deposited in a patient (integral dose) for a given target dose is always

10 lower in comparison to conventional radiotherapy techniques based on photon beams. This is in

11 part due to the lower entrance dose and in part to the minimal exit dose. Furthermore, the sharp

12 dose gradients allow a more precise definition of the target volume, making hadrontherapy

13 suitable for treating tumours located in proximity of critical organs (i.e., chondrosarcomas of the

14 skull base located close to the spinal cord). In addition, the increased biological effectiveness of

15 ions with respect to photons (and also to protons) allows treating radioresistant tumors like

16 sarcomas and some glandular tumours [57], [58].

17 One of the main issues in hadrontherapy is the need to know the actual path of the beam in the

18 patient and where it stops. To fully exploit the potential of hadrontherapy, prediction of the

19 particles range in patient must be as accurate as possible in the planning and delivery of the

20 treatment. Being a precision technique, hadrontherapy is highly sensitive to range uncertainties

21 [59]. The impact of an improper quantification of safety margins can be more severe than in

22 conventional radiotherapy. As clinical good practice, generous safety margins are applied to the

23 particles beam range to take into account uncertainties. There is no general rule, but for instance

24 in several proton therapy centers, the treatment planning assumes an uncertainty in proton range 
1 of $3.5 \%$ of the range plus additional $1-3 \mathrm{~mm}$ [60]. These values result in a substantial overshoot

2 of $8 \mathrm{~mm}$ for a $20 \mathrm{~cm}$ range in soft tissue.

3 Many different approaches of in vivo particle range verification and monitoring have been

4 proposed in the past years. Most of them rely on the detection of the secondary radiations exiting

5 the patient after nuclear reactions occurring along the beam path. Nuclear reactions may occur all

6 along the projectile path, until close to the Bragg peak region when the kinetic energy falls below

7 the Coulomb barrier. Therefore, secondary radiation emission is correlated to the primary ion

8 range, although the underlying hadronic interaction processes differ from the electromagnetic

9 interaction governing energy loss.

10 Monitoring systems are generally based on radiation detectors composed of scintillators 11 coupled to photodetectors like PMT and, in the latest generation, SiPMs. In particular, fast timing, 12 compactness and design flexibility made the latter ones suitable for the most demanding in-beam 13 monitoring systems; in fact, they must be located in close proximity to the patient, operated during

14 the irradiation without hampering the patient bed or the gantry motion and must sustain the rate 15 of events occurring during a therapeutical session [61].

16 The most consolidated among the monitoring techniques is Positron Emission Tomography (PET)

17 [2], which allows measuring the activity of positron $\left(\beta^{+}\right)$radionuclides produced by nuclear beam 18 interactions with the tissues [62-64]. The accuracy of the treatment is controlled by comparing 19 the measured PET image with a reference image obtained by Monte Carlo simulation or by 20 intercomparing a previous fraction of the same treatment.

21 In the most advanced in-beam implementation, dual head PET cameras (rotating or stationary) 22 are positioned close to the beam nozzle or integrated in the gantry. Shao and collaborators [65] 23 first developed a PET camera for on-line image-guided adaptive proton therapy with dual rotating 24 depth-of-interaction measurable detector panels by using SiPM (here called solid-state $-17-$ 
1 photomultiplier SSPM fabricated by SensL) arrays and LYSO scintillator. The system, with 44

$2 \mathrm{~mm}$ diameter transaxial and $30 \mathrm{~mm}$ axial $\mathrm{FoV}$, was tested at the protontherapy center of the

3 University of Texas MD Anderson Cancer Center (Houston, TX) with polymethyl methacrylate

4 (PMMA) phantoms. Tomographic images were acquired including data both during and 5 min

5 after irradiations. The results show a fast converging of activity-ranges measured by the prototype

6 PET with high sensitivity and uniform resolution. Sub-mm activity-ranges were achieved with

7 minimal $6 \mathrm{~s}$ acquisition time and three spill irradiations.

8 Small scale prototypes, still featuring dual head design, rely on the digital SiPM $[28,66]$ to

9 implement in-beam TOF-PET. The PET detector comprises two DPC modules from Philips

10 coupled to LYSO pixilated crystals. The surface area was of $66 \mathrm{~mm} \times 66 \mathrm{~mm}$ [67]. Experimental

11 tests on PMMA phantoms at the Heidelberg Ion Therapy synchrotron source measured

12 uncertainty in the activity range of $0.2-0.3 \mathrm{~mm}$.

13 Dendooven and coll. [68] have made an experiment with bare digital SiPMs to test the radiation

14 hardness of such devices in a geometry configuration typical of in-beam PET operation. They

15 found that the dark count rate (DCR) became too large for successful operation after the

16 equivalent of a few weeks in a proton therapy treatment room (about $5 \times 10^{13}$ protons. This

17 corresponds to an irradiation of $10^{8} 1 \mathrm{MeV}$ equivalent neutron $/ \mathrm{cm}^{2}$, in agreement with other

18 observations on analog SiPMs [69-71], suggesting that the damage occurs in the analog sensor

19 part and not in the electronics integrated in the chip. Being radiation hardness a potential

20 showstopper for SiPM application in hadrontherapy, more studies are required to address it in a

21 typical in-beam application and to apply mitigation strategies.

22 Besides the detection of $\beta^{+}$radionuclides produced by target or projectile nuclear fragmentation,

23 the prompt emission of high-energy photons is expected to provide an instantaneous vision of the

24 beam range in matter with respect to PET [72]. The idea to use prompt-gamma (PG) detection to 
1 monitor ion ranges in hadrontherapy was first proposed in 2003 by Stichelbaut and Jongen at the

2 PTCOG meeting in San Francisco. Then a proof of principle was published by Min et al. in 2006

3 [73], with a collimated and shielded detector scanning proton beam ranges in a water tank. Testa

4 et al. showed soon after that carbon ion range is also measurable, provided Time-of-Flight (TOF)

5 is employed to discriminate PGs issued from the target from a large background of secondary

6 radiation [74]. Since then, the topic motivated many research groups worldwide, and several

7 different detection modalities were proposed.

8 The most advanced PG imaging device has been developed by IBA [75] and it is based on a single

9 slit $\mathrm{W}$ collimator of the knife-edge type to realize the 1-D projection of the beam path along the

10 beam axis. The detection part consists of scintillation detector slabs (LYSO) read out via silicon

11 photomultipliers [76]. Experiments were performed with 100, 160 and $230 \mathrm{MeV}$ protons

12 impinging on homogeneous PMMA targets. A precision $(2 \sigma)$ in shift retrieval of $4 \mathrm{~mm}$ was

13 reached. In inhomogeneous targets with inserts of tissue equivalent material, for most cases range

14 deviations can be detected within less than $2 \mathrm{~mm}$ [77].

15 Prompt gamma-ray imaging with a knife-edge camera has been applied for the first time clinically

16 for a treatment of a head and neck tumor [78]. The measured inter-fractional global range

17 variations were in the order of $\pm 2 \mathrm{~mm}$. This agrees with CT information (variations on the order

18 of $1.5 \mathrm{~mm})$.

19 Besides the previously described system with passive collimation, electronic collimation, as in

20 the Compton cameras, is used with the advantage of a higher detection efficiency. Compton

21 cameras for medical imaging have first been proposed in [79]. This type of cameras is also well

22 adapted for the purpose of prompt gamma-ray detection, since the relevant energy range of several

$23 \mathrm{MeV}$ Compton scattering is the dominant process. 
1 Llosá et al. built a prototypal camera with a monolithic $\mathrm{LaBr}_{3}$ crystal coupled by to a SiPM-array

2 for performance tests [80], including the 3-D reconstruction of the impact position in the detector

3 [81]. The final Compton camera consists of two layers of monolithic $\mathrm{LaBr}_{3}$ crystals with entrance

4 surfaces of $27.2 \times 26.8 \mathrm{~mm}^{2}$ and $32 \times 36 \mathrm{~mm}^{2}$ and thicknesses of 5 and $10 \mathrm{~mm}$, respectively [82].

5 After successful reconstruction of point-like sources, tests at a $150 \mathrm{MeV}$ proton beam showed that

6 with the reconstructed Bragg peaks shifts within $10 \mathrm{~mm}$ can be observed [83].

7 In the case of ions heavier than protons, light secondary charged fragments may be produced at

8 typically the same velocity as the projectiles, or even faster, and keep some memory of the initial

9 interaction point within the projectile range, thus providing information of the primary ion range.

10 The detection of these secondary charged particle for the verification of carbon-ion therapy is

11 sometimes called Interaction Vertex Imaging (IVI) [84-86]. Large acceptance detectors, made of

12 scintillating fibers coupled with silicon photomultipliers, enabled the observations of fragments

13 at large angles and the reconstruction of the emission vertex in homogeneous and heterogenous

14 PMMA-air phantoms [87-89].

15 The most innovative approach on range monitoring relies on hybrid detection scheme that offers

16 the unique combination of complementary information from different modalities. INSIDE

17 (Innovative SolutIons for Dosimetry in hadronthErapy) is an innovative bimodal imaging system

18 providing a robust range verification and monitoring during particle therapy treatments [90]. The

19 INSIDE bimodal system (Figure 8) consists of an in-beam PET scanner and of a tracking system

20 for IVI, called dose profiler (DP). PET is made of two planar opposite heads, $60 \mathrm{~cm}$ one to the

21 other, each composed by 10 (array of $2 \times 5$ ) detection modules. A detection module is an array of

$2216 \times 16$ LFS crystals, $3 \times 3 \times 20 \mathrm{~mm}^{3}$ each, one-to-one coupled to a matching array SiPMs. The

23 head total area is $112 \mathrm{~mm}$ (transaxial) $\times 264 \mathrm{~mm}$ (axial, along the beam direction). The DP is

24 made of 8 scintillating fibers stereo-layers, that allow reconstructing the track and extrapolate the 
1 emission points of protons exiting the patient with about $90 \%$ efficiency. The $19.2 \times 19.2 \mathrm{~cm}^{2} \mathrm{DP}$

2 planes are made of square $\left(500 \times 500 \mu \mathrm{m}^{2}\right)$ multicladding fibers read out by SiPMs [91].

3

4

5

6

7 The PET system was installed and is in operation at CNAO since 2016. After the commissioning 8 phase, it has been successfully used to monitor a patient with lacrimal gland tumor in two 9 consecutive treatment sessions. Both irradiations lasted $240 \mathrm{sec}$ [92]. The patient was treated with $103.7 \times 10^{10}$ protons per field (dose $2.2 \mathrm{GyE}$ ) in the range [66.3, 144.4] MeV/u. Figure 9 shows the 11 activation maps reconstructed after $240 \mathrm{sec}$ of irradiation and additional $30 \mathrm{sec}$ of acquisition 12 after-treatment The acquisitions were also compared with the Monte Carlo simulations 13 reproducing the same set-up. Quantitative comparison of the activation isosurfaces [93] provides

14 an average distance between data and simulation of less than one $\mathrm{mm}$ with a standard deviation 15

Figure 8: The INSIDE system at CNAO. The two in-beam PET heads are above and below the patient bed. The Dose Profiler is visible in the aluminum box on the left. ) below $2 \mathrm{~mm}$.

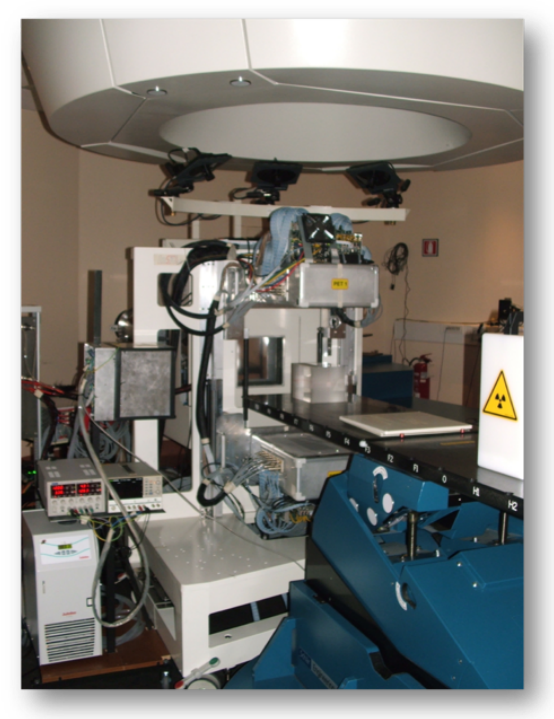




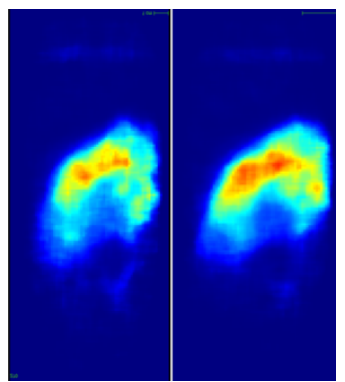

2 Figure 9: Activation maps reconstructed after 240 sec of irradiation and additional 30 sec of 3 acquisition after-treatment. Left: experimental data of the first day. Right: simulation. The beam 4 enters from top. Color map legenda: maximum intensity in red (a.u.), null intensity in blue.

5

6

The DP was completed and tested at CNAO with phantoms since the second half of 2017. Figure

1310 shows the distribution of the secondary protons generation depths along the beam direction

14 (red) as obtained irradiating an anthropomorphic phantom with $300 \mathrm{MeV} / \mathrm{u}^{12} \mathrm{C}$ ion pencil beams.

15 The distribution has been obtained with $10^{6}$ ions integrated on $1 \mathrm{~cm}^{2}$ area transversal to the beam

16 direction. Taking into account the absorption of the secondary proton in the patient tissue by

17 means of a filtering procedure [94], the emission profile (green) was calculated. The simulated emission distribution (Monte Carlo truth) is also shown for comparison (black). 
1 Another application of SiPM in hadrontherapy is proton computed tomography (pCT), i.e.,

2 imaging with energetic protons traversing the object to be reconstructed. It is a low-dose imaging

3 technique capable to improve range prediction accuracy because the particles used for imaging

4 are of the same kind as those used for treatment [95]. Furthermore, pCT may be used for

5 verification of treatment plan just before treatment.

6 Protons with known incoming energy are registered one-by-one with position-sensitive detectors

7 (trackers) before and after the object and are absorbed in an energy detector (calorimeter or range

8 counter), which thus measures residual proton energy. Whereas the tracker is usually made of

9 layers of silicon strip detectors, SiPMs are used in the latest generation of calorimeters and range

10 counters. In [96] Schulte and coll. presents a pCT system featuring a range counter, where protons

11 stop in a long stack of thin scintillating tiles, each of them working as a scintillating counter with

12 a sensitive area of $40 \times 15 \mathrm{~cm}^{2}$ using polystyrene-based scintillating tiles of $3.75 \mathrm{~mm}$ thickness

13 (or $\sim 4 \mathrm{~mm}$ when wrapped with reflecting material). The scintillating tiles were directly read out

14 by Hamamatsu MPPC (mod. S10362-33-050C) with $3 \times 3 \mathrm{~mm}^{2}$ sensitive area.

As previously observed, range monitoring devices using photons and charged particles

16 produced by the beam interacting with the patient's body have been developed, but no attempt has

17 been made yet to exploit the detection of the abundant neutron component. Since neutrons can release a significant dose far away from the tumour region, precise measurements of their flux, production energy and angle distributions are eagerly sought in order to improve the treatment 20 planning system (TPS) software. MONDO (monitor for neutron dose in hadrontherapy) is a 21 tracking detector targeting fast and ultra-fast secondary neutrons [97]. The neutron tracking 22 principle is based on the reconstruction of two consequent elastic scattering interactions of a 23 neutron with a target material. By reconstructing the recoiling protons, it is possible to measure 24 the energy and incoming direction of the neutron. Plastic scintillators are used as scattering and 25 detection media: the tracker is being developed as a matrix of squared scintillating fibers. The 
1 light produced and collected in fibers is acquired using a new type of digital SiPMs, CMOS Single

2 Photon Avalanche Diode arrays developed by Fondazione Bruno Kessler (FBK). A $4 \times 4 \times 4.8$

$3 \mathrm{~cm}^{3}$ prototype was built using $250 \mu \mathrm{m}$ thick scintillating square fibers and was tested using a

4 proton in the energy range of $60-220 \mathrm{MeV}$. The detector response has been studied, measuring

5 the energy deposited by the protons inside the fibres. Even with a non-optimised sensor it was

6 possible to identify and reconstruct the proton related tracks.

\section{6. Intraoperative Probes}

9 Intraoperative surgery based on the detection of radioactive or fluorescent tracers offers high 10 sensitivity and specificity for the discrimination of malignant tissues $[98,99]$. Counting and 11 imaging probes for medium and high-energy gamma are nowadays well established in clinical 12 routine, especially for the sentinel lymph node biopsy [100]. They include probes based on 13 semiconductor materials or on scintillators (both organic and inorganic) read-out by PMTs. New 14 developed gamma imaging probes replaces the PMT technology with SiPMs for their high signal15 to-noise ratio, compactness, low weight, simplicity of implementation and versatility.

16 In [101] authors describe a SiPM based hand-held imaging probe for intraoperative surgical 17 guidance. The camera incorporates a $\mathrm{LaBr}_{3}: \mathrm{Ce}$ scintillator plate, an array of $80 \mathrm{SiPMs}$ and a two18 layer parallel-hole collimator. The field of view is circular with a $60 \mathrm{~mm}$ diameter. The disk19 shaped camera housing is $75 \mathrm{~mm}$ in diameter, approximately $40.5 \mathrm{~mm}$ thick and has a mass of 20 only $1.4 \mathrm{~kg}$, permitting either hand-held or arm-mounted use. The gamma camera has an intrinsic 21 spatial resolution of $4.2 \mathrm{~mm}$ FWHM, an energy resolution of $21.1 \% \mathrm{FWHM}$ at $140 \mathrm{keV}$, and a 22 sensitivity of 481 and $73 \mathrm{cps} / \mathrm{MBq}$ when using the single- and double-layer collimators, 23 respectively. 
1 Goertzen and coll. [102] proposed a hand held gamma camera composed of a CsI:Tl array coupled

2 to a SensL SPMArray4M. The scintillator array is $4 \times 4$ elements directly coupled to the SiPM

3 with crystal size of $3.3 \times 3.3 \times 5 \mathrm{~mm}^{3}$. A low energy resolution parallel hole collimator with 1.2

$4 \mathrm{~mm}$ flute size, $0.2 \mathrm{~mm}$ septa and $23 \mathrm{~mm}$ depth is placed in front of the crystal array. The total

5 package comprising also the front-end electronics weighs approximately $320 \mathrm{~g}$. The hand-held

6 camera has a spatial resolution of $3.46 \mathrm{~mm}$ (at the collimator face) and a detection efficiency of

$7 \quad 162.9 \mathrm{cps} / \mathrm{MBq}$.

8 The number of positron or electron-emitting pharmaceuticals for diagnosis or therapeutic

9 purposes [103] has created a renewed interest for in situ detection of beta particles to perform real

10 time delineation of tumor boundaries [104,105]. Compared to gamma detection, the short range

11 of beta particles (around $1 \mathrm{~mm}$ in water for ${ }^{18} \mathrm{~F}$ positrons), allows a more sensitive and accurate tumor localization. Since no collimation is necessary, it is possible to design compact and light probes with a sensitivity increased by one to three orders of magnitude as compared to gamma

14 detectors. The beta particle short range also reduces the contamination from distal non-specific radiotracers uptake region, which results in an increased signal-to-noise-ratio. Spadola et al. [106] developed a simple positron probe composed of a thin scintillator covered by an optical coating and coupled through a light spreading window to a SiPM array. The photodetector is a 16channels Through Silicon Via (TSV) SiPMs array (S12642-0404PA, Hamamatsu). TSV technology allows to reduce down to $0.2 \mathrm{~mm}$ the gap in between the $3 \times 3 \mathrm{~mm}^{2}$ pixels composed of $50 \times 50 \mu \mathrm{m}^{2}$ size cells. Four SiPM arrays were assembled together on a printed circuit board

21 in order to obtain a FoV of $25.7 \times 25.7 \mathrm{~mm}^{2}$ (space in-between the arrays of $0.5 \mathrm{~mm}$ ). The imaging 22 probe configuration composed by a $0.1 \mathrm{~mm}$ thick p-terphenyl scintillator coupled to a $2 \mathrm{~mm}$ thick 23 light guide offers promising low gamma sensitivity while maintaining high beta sensitivity and 24 submillimetric intrinsic resolution over its usable field of view. 
1 Selverstone and coll. proposed in 1949 [107] the use of pure $\beta^{-}$radiation that penetrates only a

2 few millimetres of tissue with essentially no gamma contamination, being the bremsstrahlung

3 contribution, with a $0.1 \%$ emission probability, negligible. Additionally, a $\beta^{-}$probe, detecting

4 electrons and operating with lower background, would provide a clearer delineation of margins

5 of the radioactive tissue, requiring administration of a radio-pharmaceutical with a lower activity

6 and it would be smaller and easier to handle in the surgical environment. The idea was soon

7 abandoned at the time, but the developments of new radio-tracers and specific intra-operative

8 probes renovated the interest for this application. In [108] the authors reports of a $\beta^{-}$probe

9 prototype composed by a p-terphenil scintillator cylinder of $10 \mathrm{~mm}$ in diameter, $3 \mathrm{~mm}$ in height,

10 surrounded by a $5.4 \mathrm{~mm}$ layer of PVC. The front face is covered by a $10 \mu \mathrm{m}$ layer of Aluminum.

11 In this prototype, the scintillation light is collected by a SiPM (B. Series 10035 by SensL) directly

12 coupled to the back of the scintillator, with an active area of $1 \times 1 \mathrm{~mm}^{2}$. Feasibility studies for

13 meningioma, glioma and neuroendocrine tumors were performed assuming administration of

14 90Y-DOTATOC (a somatostatine analogue). They showed excellent agreement between

15 experimental and expected rates for lesions and healthy tissues: e.g. the bulk tumor showed

16 signals of $\sim 100 \mathrm{cps}, 0.2 \mathrm{~mL}$ residual signals of $\sim 40 \mathrm{cps}$ and healthy tissues of less than $5 \mathrm{cps}$.

17 Compactness and miniaturization are essential requirements for endoscopic probes and

18 SiPMs are the ideal solution also for this application. SiPMs have been employed in the

19 EndoTOFPET-US [109] endoscopic probe for prostate imaging. The EndoTOFPET-US detector

20 is being developed for the evaluation of new biomarkers and for diagnostic interventional

21 imaging. It consists of a miniaturized PET detector head mounted on an ultrasound endoscope as

22 well as an external detector plate being located next to the patient [110]. The detection of the $\beta^{+}$

23 annihilation $\gamma$-rays is carried out with scintillation crystals read out by SiPM. The endoscopic PET

24 detector is mounted on the trans-rectal ultrasound (US) endoscope EUP-U533 from Hitachi. The

25 PET head extension is clamped onto the endoscope. The PET head must not exceed a volume of $-26-$ 
$123 \times 23 \times 40 \mathrm{~mm}^{3}$ due to anatomical constraints. Due to the immediate proximity of the detector

2 to the organ under study, a high granularity is crucial for an excellent spatial resolution. This is

3 realized by the use of two matrices of $9 \times 18$ LYSO scintillator crystals from Proteus with a size

4 of $0.71 \times 0.71 \times 15 \mathrm{~mm}^{3}$ read-out by fully digital SiPMs (96).

\section{7. Conclusions and Future Outlook}

6 The SiPM found a fertile ground for applications in medicine since the very beginning [111] and

7 in particular for PET. Notwithstanding the many developments and proof of principle

8 experiments, very few apparatuses based on SiPM succeeded in reaching the market. In fact, once

9 a medical device has hit the market is going to stay for 10-20 years before a new type of device

10 takes his place. This is especially true for very expensive medical imaging devices for which the

11 industry wants to fully recover its investment and to make profit before launching a new one. In

12 the end, very few PET-only or PET/CT systems are SiPM based and this happened mostly for

13 implementing the TOF-PET option that had a major step forward based on this technology (see

14 section 3).

15 The turning point for SiPM deployment in medicine was the development of PET/MR systems.

16 When the clinical indications appeared to suggest a better diagnosis and prognosis for various

17 illnesses by the use of a PET/MR instead of a PET/CT [46,112], the SiPM became mandatory

18 because of the incompatibility of the PMTs with the magnetic field and the better performance

19 with respect to APD (see section 4). Although the SiPM are fully compatible with the magnetic

20 field the research has concentrated on obtaining the full compatibility of PET electronics with

21 MRI and of MRI RF sequences with PET data acquisition. This new technological problem has

22 found various solutions, but not yet a unique recipe to solve the problem entirely [113,114].

23 Furthermore, because of the variation of the SiPM gain and consequently of increasing dark noise

24 with temperature, the SiPM and the analog/digital electronics inside the magnet must be cooled 
1 and kept under controlled temperature. Various methods have been used: air cooling, water

2 cooling, cold liquid cooling [115]. Hence the technology complexity has been transferred for the

3 original "block detector" of 1986 [4] to the distributed digital electronics and packaging

4 A successful field of SiPM applications has recently been that of range monitoring in

5 hadrontherapy (see section 5). However, in this case the critical issue of radiation damage of the

6 SiPM must still be investigated in depth. It is expected that in the future SiPM could also have a

7 role in replacing imaging devices in radiotherapy. Other fields where it is expected to be a high

8 demand of SiPMs are biosensor devices [116] and point of care applications at large. Thanks to

9 the small size and low cost, the SiPM could really represent the enabling technology for these

10 fields that are rapidly emerging in the medical sector.

11 It is expected the new generation of SiPM will be in CMOS technology with electronics on chip

12 [117]. This development will be primarily driven by the impact of SiPM on industrial processes,

13 safety, homeland security, automotive applications and more. Once transferred to medicine it will

14 produce a big step forward in diagnosis, prognosis and therapy based on more and more

15 quantitative medical imaging procedures.

17 Bibliography

18 [1] R. Hofstadter, Means for Detecting Ionizing Radiations, US2585551A, n.d.

19 [2] Del Guerra, A., N. Belcari, N., M. Bisogni, Riv. Nuovo Cimento 39 (2016) 155-223.

20 [3] M. Conti, Phys. Med. 25 (2009) 1-11.

21 [4] M.E. Casey, R. Nutt, IEEE Trans. Nucl. Sci. 33 (1986) 460-463.

22 [5] A. Del Guerra, G. Di Domenico, M. Scandola, G. Zavattini, IEEE Trans. Nucl. Sci. 2345 (1998) 3105-3108.

24 [6] S.R. Cherry, Y. Shao, R.W. Silverman, K. Meadors, S. Siegel, A. Chatziioannou, J.W. Young, W. Jones, J.C. Moyers, D. Newport, A. Boutefnouchet, T.H. Farquhar, M. Andreaco, M.J. Paulus, D.M. Binkley, R. Nutt, M.E. Phelps, IEEE Trans. Nucl. Sci. 44 (1997) 1161-1166.

[7] C. Casella, M. Heller, C. Joram, T. Schneider, Nucl. Instrum. Methods Phys. Res. Sect. Accel. Spectrometers Detect. Assoc. Equip. 736 (2014) 161-168. 
[8] A.J. Gonzalez, A. Aguilar, P. Conde, L. Hernandez, L. Moliner, L.F. Vidal, F. Sanchez, S. Sanchez, C. Correcher, C. Molinos, J. Barbera, K. Lankes, S. Junge, T. Bruckbauer, P. Bruyndonckx, J.M. Benlloch, IEEE Trans. Nucl. Sci. 63 (2016) 2471-2477.

[9] J. Du, X. Bai, A. Gola, F. Acerbi, A. Ferri, C. Piemonte, Y. Yang, S.R. Cherry, Phys. Med. Biol. 63 (2018) 035035.

[10] H. Zaidi, A. Del Guerra, Med. Phys. 38 (2011) 5667-5689.

[11] B.J. Pichler, H.F. Wehrl, A. Kolb, M.S. Judenhofer, Semin. Nucl. Med. 38 (2008) 199-208.

[12] J.J. Vaquero, J.J. Sánchez, J.M. Udías, J. Cal-González, M. Desco, Nucl. Instrum. Methods Phys. Res. Sect. Accel. Spectrometers Detect. Assoc. Equip. 702 (2013) 83-87.

[13] R.S. Ohl, Light-Sensitive Electric Device Including Silicon, US Patent 2443542, 1948.

[14] J. Nishizawa, P-I-N Photo-Diode, 1952.

[15] C. Piemonte, R. Battiston, M. Boscardin, G.-F. Dalla Betta, A. Del Guerra, N. Dinu, A. Pozza, N. Zorzi, IEEE Trans. Nucl. Sci. 54 (2007) 236-244.

[16] M.G. Bisogni, M. Morrocchi, Nucl. Instrum. Methods Phys. Res. Sect. Accel. Spectrometers Detect. Assoc. Equip. 809 (2016) 140-148.

[17] D.J. Herbert, S. Moehrs, N. D’Ascenzo, N. Belcari, A. Del Guerra, F. Morsani, V. Saveliev, Nucl. Instrum. Methods Phys. Res. Sect. Accel. Spectrometers Detect. Assoc. Equip. 573 (2007) 84-87.

[18] G. Llosá, R. Battiston, N. Belcari, M. Boscardin, G. Collazuol, F. Corsi, G.-. Dalla Betta, A. Del Guerra, N. Dinu, G. Levi, S. Marcatili, S. Moehrs, C. Marzocca, C. Piemonte, A. Pozza, IEEE Trans. Nucl. Sci. 55 (2008) 877-881.

[19] R. Lecomte, J. Cadorette, S. Rodrigue, D. Lapointe, D. Rouleau, M. Bentourkia, R. Yao, P. Msaki, IEEE Trans. Nucl. Sci. 43 (1996) 1952-1957.

[20] D.P. McElroy, M. Hoose, W. Pimpl, V. Spanoudaki, T. Schüler, S.I. Ziegler, Phys. Med. Biol. 50 (2005) 3323-3335.

[21] B.J. Kemp, C.B. Hruska, A.R. McFarland, M.W. Lenox, V.J. Lowe, Phys. Med. Biol. 54 (2009) 2359-2376.

[22] I. Szanda, J. Mackewn, G. Patay, P. Major, K. Sunassee, G.E. Mullen, G. Nemeth, Y. Haemisch, P.J. Blower, P.K. Marsden, J. Nucl. Med. 52 (2011) 1741-1747.

[23] M.Z. Pajak, D. Volgyes, S.L. Pimlott, C.C. Salvador, A.S. Asensi, C. McKeown, J. Waldeck, K.I. Anderson, Open Med. J. 3 (2016) 12-26.

[24] S. Krishnamoorthy, E. Blankemeyer, P. Mollet, S. Surti, R. Van Holen, J.S. Karp, Phys. Med. Biol. 63 (2018) 155013.

[25] S. Vandenberghe, E. Mikhaylova, E. D’Hoe, P. Mollet, J.S. Karp, EJNMMI Phys. 3 (2016).

[26] S. Gundacker, E. Auffray, B. Frisch, P. Jarron, A. Knapitsch, T. Meyer, M. Pizzichemi, P. Lecoq, J. Instrum. 8 (2013) P07014-P07014.

[27] D.R. Schaart, E. Charbon, T. Frach, V. Schulz, Nucl. Instrum. Methods Phys. Res. Sect. Accel. Spectrometers Detect. Assoc. Equip. 809 (2016) 31-52.

[28] T. Frach, G. Prescher, C. Degenhardt, R. de Gruyter, A. Schmitz, R. Ballizany, in: IEEE, 2009, pp. 1959-1965. 
[29] D.F.C. Hsu, E. Ilan, W.T. Peterson, J. Uribe, M. Lubberink, C.S. Levin, J. Nucl. Med. 58 (2017) 1511-1518.

[30] K. Wagatsuma, K. Miwa, M. Sakata, K. Oda, H. Ono, M. Kameyama, J. Toyohara, K. Ishii, Phys. Med. 42 (2017) 203-210.

[31] S. Mandai, V. Jain, E. Charbon, in: 2012 Proc. ESSCIRC ESSCIRC, IEEE, Bordeaux, France, 2012, pp. 89-92.

[32] A.J. Lucas, R.C. Hawkes, R.E. Ansorge, G.B. Williams, R.E. Nutt, J.C. Clark, T.D. Fryer, T.A. Carpenter, Technol. Cancer Res. Treat. 5 (2006) 337-341.

[33] M.S. Judenhofer, C. Catana, B.K. Swann, S.B. Siegel, W.-I. Jung, R.E. Nutt, S.R. Cherry, C.D. Claussen, B.J. Pichler, Radiology 244 (2007) 807-814.

[34] S.J. Hong, H.G. Kang, G.B. Ko, I.C. Song, J.-T. Rhee, J.S. Lee, Phys. Med. Biol. 57 (2012) 3869-3883.

[35] N. Omidvari, J. Cabello, G. Topping, F.R. Schneider, S. Paul, M. Schwaiger, S.I. Ziegler, Phys. Med. Biol. (2017).

[36] R. Becker, A. Buck, C. Casella, G. Dissertori, J. Fischer, A. Howard, M. Ito, P. Khateri, W. Lustermann, J.F. Oliver, U. Röser, G. Warnock, B. Weber, Nucl. Instrum. Methods Phys. Res. Sect. Accel. Spectrometers Detect. Assoc. Equip. 845 (2017) 648-651.

[37] S.H. Maramraju, S.D. Smith, S.S. Junnarkar, D. Schulz, S. Stoll, B. Ravindranath, M.L. Purschke, S. Rescia, S. Southekal, J.-F. Pratte, P. Vaska, C.L. Woody, D.J. Schlyer, Phys. Med. Biol. 56 (2011) 2459-2480.

[38] A. Nassalski, M. Moszynski, A. Syntfeld-Kazuch, T. Szczesniak, L. Swiderski, D. Wolski, T. Batsch, J. Baszak, IEEE Trans. Nucl. Sci. 57 (2010) 1008-1014.

[39] K.J. Hong, Y. Choi, J.H. Jung, J. Kang, W. Hu, H.K. Lim, Y. Huh, S. Kim, J.W. Jung, K.B. Kim, M.S. Song, H. Park, Med. Phys. 40 (2013) 042503.

[40] A.M. Grant, T.W. Deller, M.M. Khalighi, S.H. Maramraju, G. Delso, C.S. Levin, Med. Phys. 43 (2016) 2334-2343.

[41] B. Weissler, P. Gebhardt, P. Duppenbecker, B. Goldschmidt, A. Salomon, D. Schug, J. Wehner, C. Lerche, D. Wirtz, W. Renz, K. Schumacher, B. Zwaans, P. Marsden, F. Kiessling, V. Schulz, in: 2012 IEEE Nucl. Sci. Symp. Med. Imaging Conf. Rec. NSSMIC, IEEE, Anaheim, CA, USA, 2012, pp. 2113-2116.

[42] B. Weissler, P. Gebhardt, P.M. Dueppenbecker, J. Wehner, D. Schug, C.W. Lerche, B. Goldschmidt, A. Salomon, I. Verel, E. Heijman, M. Perkuhn, D. Heberling, R.M. Botnar, F. Kiessling, V. Schulz, IEEE Trans. Med. Imaging 34 (2015) 2258-2270.

[43] D. Schug, J. Wehner, P.M. Dueppenbecker, B. Weissler, P. Gebhardt, B. Goldschmidt, A. Salomon, F. Kiessling, V. Schulz, Phys. Med. Biol. 60 (2015) 7045-7067.

[44] D. Schug, C. Lerche, B. Weissler, P. Gebhardt, B. Goldschmidt, J. Wehner, P.M. Dueppenbecker, A. Salomon, P. Hallen, F. Kiessling, V. Schulz, Phys. Med. Biol. 61 (2016) 2851-2878.

[45] H. Herzog, K.-J. Langen, C. Weirich, E. Rota Kops, J. Kaffanke, L. Tellmann, J. Scheins, I. Neuner, G. Stoffels, K. Fischer, L. Caldeira, H.H. Coenen, N.J. Shah, Nuklearmedizin 50 (2011) 74-82. 
[46] D.L. Bailey, B.J. Pichler, B. Gückel, H. Barthel, A.J. Beer, R. Botnar, R. Gillies, V. Goh, M. Gotthardt, R.J. Hicks, R. Lanzenberger, C. la Fougere, M. Lentschig, S.G. Nekolla, T. Niederdraenk, K. Nikolaou, J. Nuyts, D. Olego, K.A.. Riklund, A. Signore, M. Schäfers, V. Sossi, M. Suminski, P. Veit-Haibach, L. Umutlu, M. Wissmeyer, T. Beyer, Mol. Imaging Biol. 18 (2016) 637-650.

[47] (n.d.).

[48] J.M. Benlloch, A.J. González, R. Pani, E. Preziosi, C. Jackson, J. Murphy, J. Barberá, C. Correcher, S. Aussenhofer, D. Gareis, D. Visvikis, J. Bert, B. Langstrom, L. Farde, M. Toth, J. Haggkvist, F.V. Caixeta, K. Kullander, I. SomlaiSchweiger, M. Schwaiger, Eur. Psychiatry 50 (2018) 21-27.

[49] (n.d.).

[50] A. Del Guerra, S. Ahmad, M. Avram, N. Belcari, A. Berneking, L. Biagi, M.G. Bisogni, F. Brandl, J. Cabello, N. Camarlinghi, P. Cerello, C.-H. Choi, S. Coli, S. Colpo, J. Fleury, V. Gagliardi, G. Giraudo, K. Heekeren, W. Kawohl, T. Kostou, J.-L. Lefaucheur, C. Lerche, G. Loudos, M. Morrocchi, J. Muller, M. Mustafa, I. Neuner, P. Papadimitroulas, F. Pennazio, R. Rajkumar, C.R. Brambilla, J. Rivoire, E.R. Kops, J. Scheins, R. Schimpf, N.J. Shah, C. Sorg, G. Sportelli, M. Tosetti, R. Trinchero, C. Wyss, S. Ziegler, Eur. Psychiatry 50 (2018) 7-20.

[51] G. Sportelli, S. Ahmad, N. Belcari, M.G. Bisogni, N. Camarlinghi, A. Di Pasquale, S. Dussoni, J. Fleury, M. Morrocchi, E. Zaccaro, A. Del Guerra, IEEE Trans. Radiat. Plasma Med. Sci. 1 (2017) 168-177.

[52] Y. Liang, H. Peng, Nucl. Instrum. Methods Phys. Res. Sect. Accel. Spectrometers Detect. Assoc. Equip. 751 (2014) 23-30.

[53] (n.d.).

[54] M. Carminati, G.L. Montagnani, M. Occhipinti, A. Kuehne, T. Niendorf, K. Nagy, A. Nagy, M. Czeller, C. Fiorini, IEEE Trans. Radiat. Plasma Med. Sci. 2 (2018) 369-379.

[55] Https://Www.Ptcog.Ch/. Accessed 7 August 2018, n.d.

[56] R.R. Wilson, Radiology 47 (1946) 487-491.

[57] M. Durante, R. Orecchia, J.S. Loeffler, Nat. Rev. Clin. Oncol. 14 (2017) 483-495.

[58] M. Durante, H. Paganetti, Rep. Prog. Phys. 79 (2016) 096702.

[59] A.-C. Knopf, A. Lomax, Phys. Med. Biol. 58 (2013) R131-R160.

[60] H. Paganetti, Phys. Med. Biol. 57 (2012) R99-117.

[61] K. Parodi, Nucl. Instrum. Methods Phys. Res. Sect. Accel. Spectrometers Detect. Assoc. Equip. 809 (2016) 113-119.

[62] K. Parodi, Med. Phys. 42 (2015) 7153-7168.

[63] G. Sportelli, N. Belcari, N. Camarlinghi, G.A.P. Cirrone, G. Cuttone, S. Ferretti, A. Kraan, J.E. Ortuño, F. Romano, A. Santos, K. Straub, A. Tramontana, A.D. Guerra, V. Rosso, Phys. Med. Biol. 59 (2014) 43-60.

[64] X. Zhu, G.E. Fakhri, Theranostics 3 (2013) 731-740.

[65] Y. Shao, X. Sun, K. Lou, X.R. Zhu, D. Mirkovic, F. Poenisch, D. Grosshans, Phys. Med. Biol. 59 (2014) 3373-3388.

[66] Y. Haemisch, T. Frach, C. Degenhardt, A. Thon, Phys. Procedia 37 (2012) 15461560 . 
[67] P. Cambraia Lopes, J. Bauer, A. Salomon, I. Rinaldi, V. Tabacchini, T. Tessonnier, P. Crespo, K. Parodi, D.R. Schaart, Phys. Med. Biol. 61 (2016) 6203-6230.

[68] F. Diblen, T. Buitenhuis, T. Solf, P. Rodrigues, E. van der Graaf, M.-J. van Goethem, S. Brandenburg, P. Dendooven, IEEE Trans. Nucl. Sci. 64 (2017) 18911896.

[69] P. Bohn, A. Clough, E. Hazen, A. Heering, J. Rohlf, J. Freeman, S. Los, E. Cascio, S. Kuleshov, Y. Musienko, C. Piemonte, Nucl. Instrum. Methods Phys. Res. Sect. Accel. Spectrometers Detect. Assoc. Equip. 598 (2009) 722-736.

[70] S. Sánchez Majos, P. Achenbach, C. Ayerbe Gayoso, J.C. Bernauer, R. Böhm, M.O. Distler, M. Gómez Rodríguez de la Paz, H. Merkel, U. Müller, L. Nungesser, J. Pochodzalla, B.S. Schlimme, T. Walcher, M. Weinriefer, C.J. Yoon, Nucl. Instrum. Methods Phys. Res. Sect. Accel. Spectrometers Detect. Assoc. Equip. 602 (2009) 506-510.

[71] T. Matsumura, T. Matsubara, T. Hiraiwa, K. Horie, M. Kuze, K. Miyabayashi, A. Okamura, T. Sawada, S. Shimizu, T. Shinkawa, T. Tsunemi, M. Yosoi, Nucl. Instrum. Methods Phys. Res. Sect. Accel. Spectrometers Detect. Assoc. Equip. 603 (2009) 301-308.

[72] J. Krimmer, D. Dauvergne, J.M. Létang, é. Testa, Nucl. Instrum. Methods Phys. Res. Sect. Accel. Spectrometers Detect. Assoc. Equip. 878 (2018) 58-73.

[73] C.-H. Min, C.H. Kim, M.-Y. Youn, J.-W. Kim, Appl. Phys. Lett. 89 (2006) 183517.

[74] E. Testa, M. Bajard, M. Chevallier, D. Dauvergne, F. Le Foulher, N. Freud, J.-M. Létang, J.-C. Poizat, C. Ray, M. Testa, Appl. Phys. Lett. 93 (2008) 093506.

[75] J. Smeets, F. Roellinghoff, D. Prieels, F. Stichelbaut, A. Benilov, P. Busca, C. Fiorini, R. Peloso, M. Basilavecchia, T. Frizzi, J.C. Dehaes, A. Dubus, Phys. Med. Biol. 57 (2012) 3371-3405.

[76] I. Perali, A. Celani, L. Bombelli, C. Fiorini, F. Camera, E. Clementel, S. Henrotin, G. Janssens, D. Prieels, F. Roellinghoff, J. Smeets, F. Stichelbaut, F.V. Stappen, Phys. Med. Biol. 59 (2014) 5849-5871.

[77] M. Priegnitz, S. Helmbrecht, G. Janssens, I. Perali, J. Smeets, F. Vander Stappen, E. Sterpin, F. Fiedler, Phys. Med. Biol. 60 (2015) 4849-4871.

[78] C. Richter, G. Pausch, S. Barczyk, M. Priegnitz, I. Keitz, J. Thiele, J. Smeets, F.V. Stappen, L. Bombelli, C. Fiorini, L. Hotoiu, I. Perali, D. Prieels, W. Enghardt, M. Baumann, Radiother. Oncol. 118 (2016) 232-237.

[79] D.B. Everett, J.S. Fleming, R.W. Todd, J.M. Nightingale, Proc. Inst. Electr. Eng. 124 (1977) 995.

[80] G. Llosá, J. Barrio, J. Cabello, A. Crespo, C. Lacasta, M. Rafecas, S. Callier, C. de La Taille, L. Raux, Nucl. Instrum. Methods Phys. Res. Sect. Accel. Spectrometers Detect. Assoc. Equip. 695 (2012) 105-108.

[81] Z. Li, M. Wedrowski, P. Bruyndonckx, G. Vandersteen, Phys. Med. Biol. 55 (2010) 6515-6532.

[82] G. Llosá, J. Cabello, S. Callier, J.E. Gillam, C. Lacasta, M. Rafecas, L. Raux, C. Solaz, V. Stankova, C. de La Taille, M. Trovato, J. Barrio, Nucl. Instrum. Methods Phys. Res. Sect. Accel. Spectrometers Detect. Assoc. Equip. 718 (2013) 130-133. 
[83] P. Solevi, E. Muñoz, C. Solaz, M. Trovato, P. Dendooven, J.E. Gillam, C. Lacasta, J.F. Oliver, M. Rafecas, I. Torres-Espallardo, G. Llosá, Phys. Med. Biol. 61 (2016) 5149-5165.

[84] U. Amaldi, R. Bonomi, S. Braccini, M. Crescenti, A. Degiovanni, M. Garlasché, A. Garonna, G. Magrin, C. Mellace, P. Pearce, G. Pittà, P. Puggioni, E. Rosso, S. Verdú Andrés, R. Wegner, M. Weiss, R. Zennaro, Nucl. Instrum. Methods Phys. Res. Sect. Accel. Spectrometers Detect. Assoc. Equip. 620 (2010) 563-577.

[85] K. Gwosch, B. Hartmann, J. Jakubek, C. Granja, P. Soukup, O. Jäkel, M. Martišíková, Phys. Med. Biol. 58 (2013) 3755-3773.

[86] P. Henriquet, E. Testa, M. Chevallier, D. Dauvergne, G. Dedes, N. Freud, J. Krimmer, J.M. Létang, C. Ray, M.-H. Richard, F. Sauli, Phys. Med. Biol. 57 (2012) 4655-4669.

[87] L. Piersanti, F. Bellini, F. Bini, F. Collamati, E. De Lucia, M. Durante, R. Faccini, F. Ferroni, S. Fiore, E. Iarocci, C.L. Tessa, M. Marafini, I. Mattei, V. Patera, P.G. Ortega, A. Sarti, C. Schuy, A. Sciubba, M. Vanstalle, C. Voena, Phys. Med. Biol. 59 (2014) 1857-1872.

[88] M. Marafini, R. Paramatti, D. Pinci, G. Battistoni, F. Collamati, E. De Lucia, R. Faccini, P.M. Frallicciardi, C. Mancini-Terracciano, I. Mattei, S. Muraro, L. Piersanti, M. Rovituso, A. Rucinski, A. Russomando, A. Sarti, A. Sciubba, E. Solfaroli Camillocci, M. Toppi, G. Traini, C. Voena, V. Patera, Phys. Med. Biol. 62 (2017) 1291-1309.

[89] I. Mattei, G. Battistoni, F. Collini, E. De Lucia, M. Durante, S. Fiore, C. La Tessa, C. Mancini-Terracciano, M. Marafini, R. Mirabelli, S. Muraro, R. Paramatti, L. Piersanti, A. Rucinski, A. Russomando, A. Sarti, C. Schuy, A. Sciubba, E. Solfaroli Camillocci, M. Toppi, G. Traini, S.M. Valle, M. Vanstalle, V. Patera, Phys. Med. Biol. 62 (2017) 8483-8494.

[90] M.G. Bisogni, A. Attili, G. Battistoni, N. Belcari, N. Camarlinghi, P. Cerello, S. Coli, A. Del Guerra, A. Ferrari, V. Ferrero, E. Fiorina, G. Giraudo, E. Kostara, M. Morrocchi, F. Pennazio, C. Peroni, M.A. Piliero, G. Pirrone, A. Rivetti, M.D. Rolo, V. Rosso, P. Sala, G. Sportelli, R. Wheadon, J. Med. Imaging 4 (2016) 011005.

[91] S. Muraro, G. Battistoni, F. Collamati, E. De Lucia, R. Faccini, F. Ferroni, S. Fiore, P. Frallicciardi, M. Marafini, I. Mattei, S. Morganti, R. Paramatti, L. Piersanti, D. Pinci, A. Rucinski, A. Russomando, A. Sarti, A. Sciubba, E. Solfaroli-Camillocci, M. Toppi, G. Traini, C. Voena, V. Patera, Front. Oncol. 6 (2016).

[92] V. Ferrero, E. Fiorina, M. Morrocchi, F. Pennazio, G. Baroni, G. Battistoni, N. Belcari, N. Camarlinghi, M. Ciocca, A. Del Guerra, M. Donetti, S. Giordanengo, G. Giraudo, V. Patera, C. Peroni, A. Rivetti, M.D. da R. Rolo, S. Rossi, V. Rosso, G. Sportelli, S. Tampellini, F. Valvo, R. Wheadon, P. Cerello, M.G. Bisogni, Sci. Rep. 8 (2018).

[93] E. Fiorina, V. Ferrero, F. Pennazio, G. Baroni, G. Battistoni, N. Belcari, P. Cerello, N. Camarlinghi, M. Ciocca, A. Del Guerra, M. Donetti, A. Ferrari, S. Giordanengo, G. Giraudo, A. Mairani, M. Morrocchi, C. Peroni, A. Rivetti, M.D. Da Rocha Rolo, S. Rossi, V. Rosso, P. Sala, G. Sportelli, S. Tampellini, F. Valvo, R. Wheadon, M.G. Bisogni, Phys. Med. 51 (2018) 71-80. 
[94] G. Traini, G. Battistoni, A. Bollella, F. Collamati, E. De Lucia, R. Faccini, F. Ferroni, P.M. Frallicciardi, C. Mancini-Terracciano, M. Marafini, I. Mattei, F. Miraglia, S. Muraro, R. Paramatti, L. Piersanti, D. Pinci, A. Rucinski, A. Russomando, A. Sarti, A. Sciubba, M. Senzacqua, E. Solfaroli-Camillocci, M. Toppi, C. Voena, V. Patera, Phys. Med. 34 (2017) 18-27.

[95] R. Schulte, V. Bashkirov, Tianfang Li, Zhengrong Liang, K. Mueller, J. Heimann, L.R. Johnson, B. Keeney, H.F.-W. Sadrozinski, A. Seiden, D.C. Williams, Lan Zhang, Zhang Li, S. Peggs, T. Satogata, C. Woody, IEEE Trans. Nucl. Sci. 51 (2004) 866-872.

[96] V.A. Bashkirov, R.P. Johnson, H.F.-W. Sadrozinski, R.W. Schulte, Nucl. Instrum. Methods Phys. Res. Sect. Accel. Spectrometers Detect. Assoc. Equip. 809 (2016) 120-129.

[97] G. Traini, G. Battistoni, V. Giacometti, E. Gioscio, M. Marafini, R. Mirabelli, D. Pinci, A. Sarti, A. Sciubba, V. Patera, J. Instrum. 13 (2018) C04014-C04014.

[98] R.A.V. OLMOS, S. VIDAL-SICART, G. MANCA, G. MARIANI, L.F. LEÓNRAMÍREZ, D. RUBELLO, F. GIAMMARILE, Q. J. Nucl. Med. Mol. Imaging (2017).

[99] D. Hellingman, L.J. de Wit-van der Veen, W.M.C. Klop, R.A.V. Olmos, Clin. Nucl. Med. 40 (2015) e11-e16.

[100] M. Tsuchimochi, K. Hayama, Phys. Med. 29 (2013) 126-138.

[101] K. Popovic, J.E. McKisson, B. Kross, S. Lee, J. McKisson, A.G. Weisenberger, J. Proffitt, A. Stolin, S. Majewski, M.B. Williams, IEEE Trans. Nucl. Sci. 61 (2014) 1084-1091.

[102] A.L. Goertzen et al., in: 2013 IEEE Nucl. Sci. Symp. Med. Imaging Conf. 2013 NSSMIC, IEEE, Seoul, Korea (South), 2013, pp. 1-4.

[103] S.J. González, L. González, J. Wong, P. Brader, M. Zakowski, M. Gönen, F. Daghighian, Y. Fong, V.E. Strong, J. Gastrointest. Surg. 15 (2011) 358-366.

[104] B. Singh, B.C. Stack, S. Thacker, V. Gaysinskiy, T. Bartel, V. Lowe, S. Cool, G. Entine, V. Nagarkar, Ann. Nucl. Med. 27 (2013) 203-208.

[105] N. Hudin, L. Pinot, N. Dinu, Y. Charon, V. Puill, B. Janvier, V. Chaumat, M.-A. Duval, D. Abi Haidar, R. Siebert, L. Ménard, Nucl. Instrum. Methods Phys. Res. Sect. Accel. Spectrometers Detect. Assoc. Equip. 695 (2012) 242-246.

[106] S. Spadola, M.-A. Verdier, L. Pinot, C. Esnault, N. Dinu, Y. Charon, M.-A. Duval, L. Ménard, J. Instrum. 11 (2016) P12019-P12019.

[107] B. Selverstone, J. Am. Med. Assoc. 140 (1949) 277.

[108] E.S. Camillocci, G. Baroni, F. Bellini, V. Bocci, F. Collamati, M. Cremonesi, E. De Lucia, P. Ferroli, S. Fiore, C.M. Grana, M. Marafini, I. Mattei, S. Morganti, G. Paganelli, V. Patera, L. Piersanti, L. Recchia, A. Russomando, M. Schiariti, A. Sarti, A. Sciubba, C. Voena, R. Faccini, Sci. Rep. 4 (2015).

[109] B. Frisch, Nucl. Instrum. Methods Phys. Res. Sect. Accel. Spectrometers Detect. Assoc. Equip. 732 (2013) 577-580.

[110] M. Zvolský, the EndoTOFPET-US Collaboration, J. Phys. Conf. Ser. 587 (2015) 012068.

[111] V. Saveliev, V. Golovin, Nucl. Instrum. Methods Phys. Res. Sect. Accel. Spectrometers Detect. Assoc. Equip. 442 (2000) 223-229. 
[112] Z. Chen, S.D. Jamadar, S. Li, F. Sforazzini, J. Baran, N. Ferris, N.J. Shah, G.F.

2 Egan, Hum. Brain Mapp. (2018).

3 [113] R.F. Muzic, F.P. DiFilippo, Semin. Roentgenol. 49 (2014) 242-254.

4 [114] S. Vandenberghe, P.K. Marsden, Phys. Med. Biol. 60 (2015) R115-R154.

5 [115] R. Dohle, I. Sacco, T. Rittweg, T. Friedrich, G. Henning, J. Goßler, P. Fischer, Int.

[116] H. Li, N. Lopes, S. Moser, G. Sayler, S. Ripp, Biosens. Bioelectron. 33 (2012) 299-303.

[117] N. D'Ascenzo, X. Zhang, Q. Xie, Sensors 17 (2017) 2204. 